Please ensure that your abstract fits into one column on one page and complies with the Instructions to Authors available from the Abstract Submission web page.

\title{
Shipboard trace metal determinations using micro- Sequential Injection Lab on Valve methodology
}

\author{
HATTA, M., MASURES, C.I. AND RUZICKA, J.
}

University of Hawaii, 1000 Pope Road, Honolulu, HI, 96822.

USA.mhatta@hawaii.edu; gweiss@hawaii.edu;

measures@hawaii.edu

The importance of hydrothermal iron $(\mathrm{Fe})$ in global marine productivity has only recently been recognized (Tagliabue et al., Nature 543, 51-59, 2017). This is because hydrothermal $\mathrm{Fe}$ was believed to precipitate quantitatively near the vent site. Recent findings in the Pacific Oecan, however, shows the basin-scale transport of hydrothermal dissolved metals across the South Pacific Ocean (Resing et al., Nature 523, 203-206, 2015). Futhermore, Fitzsimmons et al., (Nature Geoscience 10, 195-201, 2017) suggests the imporatnce of the balance between stabilization in the dissolved phase and the reversibility of exchange onto sinking particles within the hyderothermal plume.

Currently our understanding of biogeochemical processes in the ocean is limited by our ability to obtain extensive data sets in a variety of hydrographic settings and over a range of pertinent time scales. While significant progress has been made in the development of reliable nitrate, oxygen and carbonate parameters sensors which are now being incorporated into the Argo global array and at time-series sites, the bulk of the data for other important biogeochemical variables (phosphate, silicate and trace metals) still relies primarily on instrumentation designed 60 years ago. Therefore, a pressing need is to develop more advanced technologies for trace metal determinations. A promising avenue of research is the miniaturization and automation of established chemical assays for trace metals using microfluidics.

In our work towards this goal, we have developed miniaturized automated microfluidic analyzers, which through their robustness, small size, minimal reagent use $(<$ $1 \mathrm{~mL}$ per sample), and computer controlled microfluidic manipulations can meet the prerequisites for unattended operation. In this contribution, in order to determine the hydrothermal plume samples, we will discuss the application of the new technology, named programmable flow injection (pFI), to automated $\mathrm{Fe}$ determination using the various lengths of flowcells and Liquid Waveguide Capillary Flow Cell (LWCC). 